\title{
Comparing vocal structures of the parasitic and nonparasitic groups in Cuculinae
}

Heeyoung Kim, Jin-Won Lee* (10 and Jeong-Chil Yoo

\begin{abstract}
Background: Avian brood parasitism is a breeding strategy in which brood parasites lay their eggs in the nest of other species. This behavior is closely related to aspects of the biological evolution of the parasitic species such as reduced female body size and plumage color polymorphism. However, not much is known whether it is associated with the evolution of vocalization.

Methods: We collected samples of the typical male calls of 67 species belonging to the sub-family Cuculinae. Using the calls, we measured five acoustic parameters for each samples to test the differences in vocal structures between parasitic and nonparasitic species. To control for potential phylogenetic effects, we also performed phylogenetic independent contrast analyses.
\end{abstract}

Results: We found that vocal structures were relatively similar among the parasitic species with a tendency to simple and low-frequency calls. In addition, harmonic structures were observed more frequently in the nonparasitic group.

Conclusions: Overall, these results support the idea that brood parasitic behavior with associated ecological conditions may play a role in vocal evolution, a better understanding of which may greatly improve our knowledge of vocal diversification in non-oscine birds.

Keywords: Brood parasitism, Cuculinae, Non-oscine bird, Vocal evolution

\section{Background}

Hearing the sound, 'cu-coo', a simple but charismatic call produced by the Common Cuckoo, Cuculus canorus, indicates the arrival of spring to people who live in the northern hemisphere of the Eurasian continent. However, it also indicates imminent fitness loss to the family of other bird species (hosts) in the area, because they will soon lose all their progeny while gaining an alien cuckoo chick in their nests. The evolutionary arms race between avian brood parasites and their hosts to maximize their respective fitness has been a model system for co-evolutionary studies for over a century (Davies 2000; Erritzøe et al. 2012). From these studies, we know that such a co-evolutionary relationship results in profound effects, directly or indirectly, on various aspects of the biological evolution of brood parasites, for example, egg mimicry,

*Correspondence: jwlee99@khu.ac.kr

Korea Institute of Ornithology and Department of Biology, Kyung Hee University, Seoul 02447, Republic of Korea reduced female body size and accelerated phenotypic diversification (Sorenson et al. 2003; Stoddard and Stevens 2011; Medina and Langmore 2015). However, little is known about how this behavior is associated with the vocal evolution of brood parasites.

Circumstantial evidence suggests that brood parasitic behavior could be associated with the vocal evolution of parasitic species. For example, Sorenson et al. (2003) showed that the male song types of adult indigobirds, Vidua spp., could vary according to the host species that raise them as they mimic the host's song. Fuisz and de Kort (2007) proposed that the calls of the Common Cuckoo diverge according to the types of habitats that they occupy. Similarly, some generalist brood parasitic species are known to be composed of multiple lineages of host-specific races within a species (Davies 2000), and if the host-specific divergence results in vocal divergence, we may see increased intraspecific variation in the vocal characteristics in the parasitic species. Other life history traits that appear to be derived with brood parasitism 
such as reduced body size (Medina and Langmore 2015) and increased breeding home range size (Krüger and Davies 2002), may also be associated directly or indirectly with vocal evolution. In this study, we analysed male calls produced by species belonging to the subfamily Cuculinae to explore the evolutionary patterns of vocalization between the lineage of parasitic and nonparasitic species, which may have implications for understanding the vocal evolution of non-oscine birds.

\section{Methods}

\section{Data collection}

We collected acoustic and phylogenetic data for the species belonging to the subfamily Cuculinae, in which 56 parasitic and 32 nonparasitic species are currently recognized (Payne and Sorenson 2005). Among these we obtained acoustic data for 80 species from online repositories (see below). Phylogenetic tress were generated using BirdTree (birdtree.org, http://www.birdtree.org, Jetz et al. 2012) for 76 species. As a result, both acoustic and phylogenetic data were available for 67 species (42 parasitic and 25 nonparasitic species), with which we conducted further analyses.

Acoustic samples of the typical male calls were collected from mainly Xeno-Canto (www.xeno-canto.org) and AVoCet (Avian Vocalizations Center, www.avocet. zoology.msu.edu). We referred to Payne and Sorenson (2005) to determine a typical male call type for each of the species. On average, we collected $13.57 \pm 6.19$ different high quality calls across species (see Additional file 1: Table S1). In this study, we followed the scientific names used by Payne and Sorenson (2005) because these are consistent with those used in Xeno-Canto.

\section{Acoustic data measurement}

Acoustic parameters were measured using the software Raven Pro 1.4 (Cornell Lab of Ornithology 2010). We measured five parameters for each sample: high frequency (HF), low frequency (LF), delta frequency (DF), delta time (DT), and number of notes (NN) (Fig. 1). HF and LF represent the highest and lowest pitch of selected ranges of syllables, respectively, and DF is the difference between them. DT is the time duration of the syllable, and NN, the number of notes involved in a single syllable. All definitions of parameters follow the manual of Raven Pro 1.4. (Charif et al. 2010).

\section{Statistical analysis}

Analyses were conducted in two ways, with and without phylogenetic consideration. For the original data without phylogenetic consideration, the distribution patterns of vocal parameters between the parasitic and nonparasitic groups were compared using the Kolmogorov-Smirnov tests. The Wilcoxon rank sum tests were applied to examine the median difference between the two groups. On the other hand, phylogenetic independent contrast (PIC)

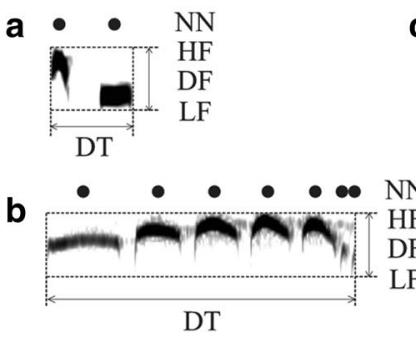

DT
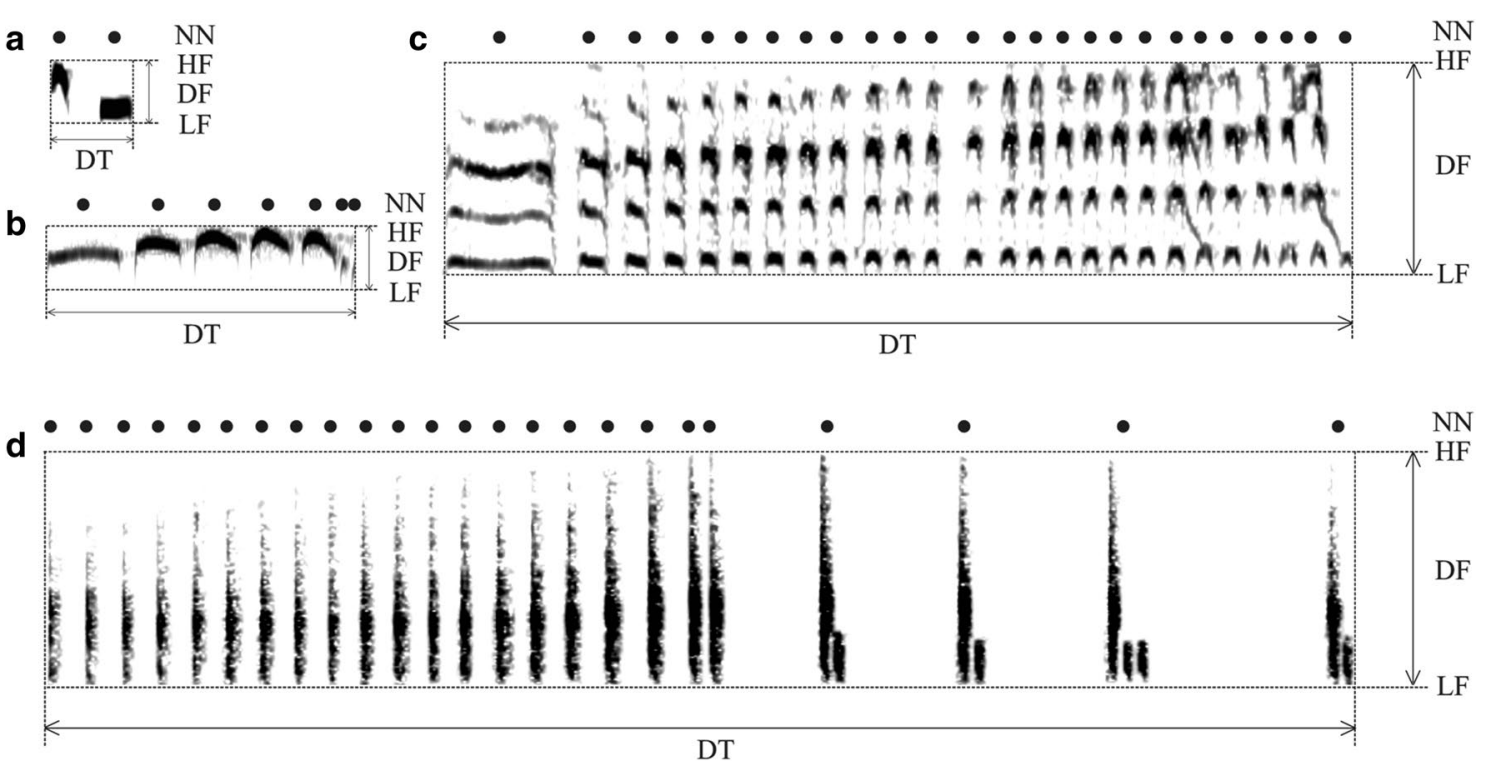

Fig. 1 Spectrograms of brood parasitic and nonparasitic species illustrating five parameters measured in this study. a Cuculus canorus and $\mathbf{b}$ Chrysococcyx caprius as brood parasitic species. Likewise, c Coccyzus minuta and d Coccyzus americanus as nonparasitic species. HF, LF, DF, DT and NN represent high frequency, low frequency, delta frequency, delta time, and the number of notes, respectively. The number of black dots above spectrograms indicates the number of notes 
analyses were conducted for each parameters using the function "brunch" in the package "Caper" (Orme et al. 2013) in R 3.2.1 (R Development Core Team 2011), which calculates the contrast values of the continuous variable on the nodes where two different categorical variables diverge. For phylogenetic information used in the analysis, we first obtained a total of 1000 possible phylogenetic trees of the 67 Cuculinae species from BirdTree (Rubolini et al. 2015), and then we randomly selected a tree from these to meet the condition of the phylogenetic tree format for the analysis (Additional file 1: Fig. S1). The choice of trees may not change the results qualitatively as shown in other studies (e.g., Medina and Langmore 2015). Statistical significance of the difference in the mean of the contrast values from zero was tested using a one-sample $t$ test. Models were checked for the robustness of the contrast using 'caic.diagnostics' function in 'Caper'. Acoustic data were averaged according to species, and log-transformed before the analysis. All statistical analyses were performed in R 3.2.1 (R Development Core Team 2011).

\section{Results}

The original data exhibited that the nonparasitic group showed larger variation in DF $(D=0.465, p=0.002)$, DT $(D=0.352, p=0.04)$, HF $(D=0.377, p=0.02)$, and $\mathrm{NN}(D=0.465, p=0.002)$. LF was the exception in that the parasitic group showed larger variation $(D=0.762$, $p<0.001$ ), albeit over small ranges (Fig. 2a-e). Likewise, harmonic structures were observed to a greater extent in the nonparasitic cuckoos (20 species out of 25) than in the parasitic group ( 7 species out of $42 ; \chi^{2}=26.8, \mathrm{df}=3$, $p<0.0001$; see Additional file 1: Table S1). As a result, the median values of vocal parameters except LF tended to be smaller in the parasitic group, although statistical significances varied among parameters (DF: $W=789$, $p<0.001$; DT: $W=548, p=0.78$; HF: $W=647, p=0.12$; LF: $W=105, p<0.001$; NN: $W=676, p=0.05)$. Consistently with the results of the original data, the phylogenetically independent contrasts test showed significant difference in vocal parameters between the parasitic and nonparasitic groups (Fig. 2f). The mean contrasts were significantly below zero for most parameters (DF: $t_{2}=-4.07, p=0.06$; DT: $t_{2}=-7.51, p=0.02$; HF: $t_{2}=-3.84, p=0.06$; NN: $t_{2}=-4.28, p=0.05$ ), indicating that the parasitic group tends to have smaller average values than the nonparasitic group for these parameters. On the other hand, the average contrasts value of LF was significantly above zero $\left(t_{2}=7.90, p=0.02\right)$, implying that the parasitic group had higher LF than the nonparasitic group, as shown with the original data.

\section{Discussion}

Our results indicate that interspecific differences in vocalization are much larger in the nonparasitic than in the parasitic group, and that the parasitic species of Cuculinae tend to have simpler and lower-frequency calls than the nonparasitic species. This imply that vocal evolution occurs much more slowly among the parasitic species in the sub-family Cuculinae with the acoustic structures converging to be simple with lowerfrequency ranges, overall suggesting the potential association between brood parasitic behavior and vocal evolution. The reason for the difference in the spectral features of the call between the two groups remain unknown. Krüger and Davies (2002) suggested that parasitic species appear to have an increased breedingrange size in the family Cuculinae, and the intraspecific vocal competition is intensive in the parasitic cuckoos (J.-W. L. unpublished data). In these situations, simple and low-frequency calls may facilitate their ability to manage relatively large breeding ranges. Ecological characteristics of breeding area such as habitat openness and humidity could also favour low-frequency calls (McCracken and Sheldon 1997; Bertelli and Tubaro 2002; Brumm 2004). Unlike the nonparasitic species, many of the parasitic species belonging to the family Cuculidae migrate from a humid tropical zone to a relatively dry temperate zone to breed, and they tend to occupy open habitats (Krüger and Davies 2002). These ecological conditions may select for lower-frequency calls with simple structures in the parasitic species. However, selection pressures that generate vocal differences between the two groups and the role of brood parasitism in there are major issues that remain to be resolved. It would be worthwhile to extend the comparative analysis that we have done in this study to other parasitic lineages such as Icteridae (Fraga 2008). Experimental studies to reveal the underlying reasons for the differences that we found in this study are also necessary to verify our conclusions.

\section{Conclusions}

Overall, our results imply the probability that brood parasitism may play a role in vocal evolution, especially for males. However, it is well recognized in some parasitic species that females also produce calls during the breeding season, such as the bubbling call produced by female Common Cuckoos (Kim et al. 2017). Our knowledge on the variation in female calls and the contribution of brood parasitism to the evolution of female calls is also very limited (York and Davies 2017). Collectively, a better 

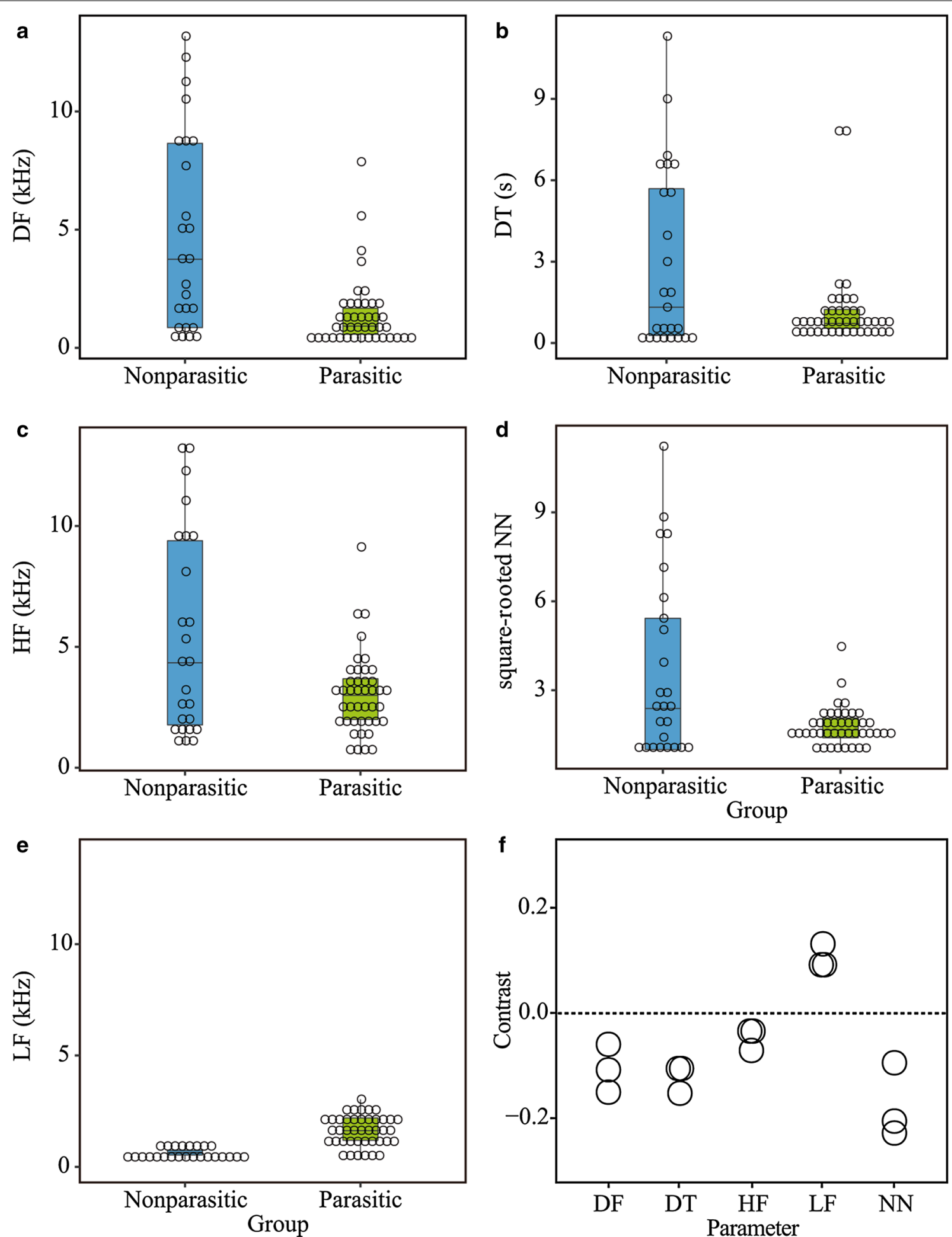

Fig. 2 Comparison of vocal parameters between the parasitic and nonparasitic groups in Cuculinae. Both the original data without phylogenetic consideration (a-e) and the result of phylogenetic independent contrasts ( $\mathbf{f}$ ) are shown. DF, DT, HF, NN, and LF represent delta frequency, delta time, high frequency, number of notes and low frequency, respectively. Each dot represents different species, and the data are summarized with box and whiskers plots 
understanding of the vocal evolution in brood parasites may greatly improve our knowledge of vocal diversification in non-oscine birds.

\section{Additional file}

Additional file 1: Table S1. A list for scientific names of 67 species, code numbers of raw files in Xeno-Canto (XC) or AVoCet (AV), the number of vocal samples obtained from each species, and the presence of harmonic structures. According to Birdlife V5, which "Birdtree.org" referred to, there are different scientific names for 11 species (see comments). Figure S1. A phylogenetic tree showing the relationship among 67 species in Cuculinae examined in this study.

\section{Authors' contributions}

HK and JWL conceived and designed the study; HK and JWL collected and analysed the data; HK, JWL and JCY wrote the manuscript. All authors read and approved the final manuscript.

\section{Acknowledgements}

We are grateful to all the people who recorded bird songs in the field and shared those through online repositories. We also thanks to Hye-Kyoung Moon for phylogenetic advice, and Hee-Jin Noh, Kyung-Hoe Kim, Ha-Na Yoo, Sung-Ho Yoon, Hae-Ni Kim, and So-Hyeon Yoo for constructive discussions about the idea of this study.

\section{Competing interests}

The authors declare that they have no competing interests.

\section{Funding}

This work was supported by Basic Science Research Program through the National Research Foundation of Korea (NRF) funded by the Ministry of Education (2012R1A6A3A04040003).

Received: 29 August 2017 Accepted: 9 October 2017

Published online: 17 October 2017

\section{References}

Bertelli S, Tubaro PL. Body mass and habitat correlates of song structure in a primitive group of birds. Biol J Linn Soc. 2002;77:423-30.
Brumm H. The impact of environmental noise on song amplitude in a territorial bird. J Anim Ecol. 2004;73:434-40.

Charif R, Strickman LM, Waack AM. Raven Pro 1.4 user's manual. New York: The Cornell Lab of Ornithology; 2010.

Cornell Lab of Ornithology. Raven: interactive sound analysis software. Version 1.4. New York: Cornell Lab of Ornithology; 2010. http://www.birds.cornell. edu/brp/Raven/Raven.html/.

Davies NB. Cuckoos, cowbirds and other cheats. London:T. \& A.D. Poyser; 2000. Erritzøe J, Mann CF, Brammer FP, Fuller RA. Cuckoos of the world. London: Christopher Helm; 2012.

Fraga RM. Phylogeny and behavioral evolution in the family Icteridae. Ornitol Neotrop. 2008;19:61-71.

Fuisz TI, de Kort SR. Habitat-dependent call divergence in the common cuckoo: Is it a potential signal for assortative mating? Proc R Soc B. 2007:274:2093-7.

Jetz W, Thomas G, Joy J, Hartmann K, Mooers A. The global diversity of birds in space and time. Nature. 2012;491:444-8.

Kim H, Lee J-W, Yoo J-C. Characteristics of female calls of four Cuculus species breeding in Korea. Kor J Ornithol. 2017;24:41-7.

Krüger O, Davies NB. The evolution of cuckoo parasitism: a comparative analysis. Proc R Soc B. 2002;269:375-81.

McCracken KG, Sheldon FH. Avian vocalizations and phylogenetic signal. Proc Natl Acad Sci USA. 1997;94:3833-6.

Medina I, Langmore NE. Coevolution is linked with phenotypic diversification but not speciation in avian brood parasites. Proc R Soc B. 2015;282:20152056.

Orme D, Freckleton R, Thomas G, Petzoldt T, Fritz S, Isaac N, Pearse W. Caper: comparative analyses of phylogenetics and evolution in R. Vienna: Comprehensive R Archive Network; 2013. http://CRAN.R-project.org/ package $=$ caper $/$.

Payne RB, Sorenson MD. The cuckoos. New York: Oxford University Press; 2005.

R Development Core Team. R: a language and environment for statistical computing. Vienna: R foundation for statistical computing; 2011. http:// www.R-project.org/.

Rubolini D, Liker A, Garamszegi LZ, Møller AP, Saino N. Using the BirdTree.org website to obtain robust phylogenies for avian comparative studies: a primer. Curr Zool. 2015;61:959-65.

Sorenson MD, Sefc KM, Payne RB. Speciation by host switch in brood parasitic indigobirds. Nature. 2003:424:928-31.

Stoddard MC, Stevens M. Avian vision and the evolution of egg color mimicry in the common cuckoo. Evolution. 2011;65:2004-13.

York JE, Davies NB. Female cuckoo calls misdirect host defences towards the wrong enemy. Nat Ecol Evol. 2017;1:1520-5.

\section{Submit your next manuscript to BioMed Central} and we will help you at every step:

- We accept pre-submission inquiries

- Our selector tool helps you to find the most relevant journal

- We provide round the clock customer support

- Convenient online submission

- Thorough peer review

- Inclusion in PubMed and all major indexing services

- Maximum visibility for your research

Submit your manuscript at www.biomedcentral.com/submit
C BioMed Central 